\title{
The outcome of IVF/ICSI cycles in male cancer patients: retrospective analysis of procedures from 2004 to 2018
}

\author{
Tanja Burnik Papler ${ }^{1}$, Eda Vrtacnik-Bokal ${ }^{1}$, Saso Drobnic ${ }^{1}$, Martin Stimpfel ${ }^{1}$ \\ ${ }^{1}$ Department of Human Reproduction, Division of Gynaecology, University Medical Centre Ljubljana, Ljubljana, Slovenia
}

Radiol Oncol 2021; 55(2): 221-228.

Received 18 August 2020

Accepted 25 January 2021

Correspondence to: Assist. Martin Stimpfel, Ph.D., Department of Human Reproduction, Division of Gynaecology, University Medical Centre Ljubljana, Slajmerjeva 3. 1000-Ljubljana, SI-Slovenia. E-mail: martin.stimpfel@gmail.com, martin.stimpfel@kclj.si

Disclosure: No potential conflicts of interest were disclosed.

Introduction. Fertility preservation is an important aspect of quality of life in oncological patients, and in men is achieved by semen cryopreservation prior to treatment. Results of in vitro fertilization (IVF) procedures in healthy infertile couples are comparable, regardless of whether fresh or cryopreserved semen is used, but are scarce in male oncological patients.

Patients and methods. We performed a retrospective analysis of IVF/intracytoplasmic sperm injection (IVF/ICSI) procedures in infertile couples where men had been treated for cancer in the past. We additionally compared the results of IVF/ICSI procedures with respect to the type of semen used (fresh, cryopreserved).

Results. We compared the success rates of 214 IVF/ICSI cycles performed in the years 2004-2018. Pregnancy $(30.0 \%$ vs. $21.4 \% ; p=0.12)$ and live-birth rates (22.3\% vs. $17.9 \% ; p=0.43$ ) per oocyte aspiration were similar between the groups in fresh cycles; however embryo utilization (48.9\% vs. $40.0 \% ; p=0.006)$ and embryo cryopreservation rates $(17.3 \%$ vs. $12.7 \% ; p=0.048)$ were significantly higher in the cryopreserved semen group. The cumulative pregnancy rate $(60.6 \%$ vs. $37.7 \% ; p=0.012)$ was significantly higher, and the live-birth rate $(45.1 \%$ vs. $34.0 \% ; p=0.21)$ non-significantly higher, in the cryopreserved semen group.

Conclusions. The success of IVF/ICSI procedures in couples where the male partner was treated for cancer in the past are the same in terms of pregnancies and live-births in fresh cycles regardless of the type of semen used. However, embryo utilization and embryo cryopreservation rates are significantly higher when cryopreserved semen is used, leading to a significantly higher cumulative number of couples who achieved at least one pregnancy.

Key words: assisted reproduction techniques; infertility; fertility preservation; sperm cryopreservation; pregnancy

\section{Introduction}

The life expectancy of cancer patients has significantly improved during the past decade due to improved oncological treatment, and it has therefore become important to enable them to have a quality life after the oncological treatment has finished. The ability to have your own biological children is one of the most important aspects of quality of life.

It is well known that oncological treatment, as well as cancer itself, can cause male infertility. Testicles are sensitive to chemotherapy and radio- therapy, which can cause a disruption of spermatogenesis. ${ }^{1,2}$ It has been estimated that approximately $15-30 \%$ of male cancer patient survivors become infertile after oncological treatment.,4 Whether or not sperm production is disrupted depends on several factors: type of cancer; semen quality prior to treatment; type, dosage and duration of oncological treatment. ${ }^{2,5}$ For instance, most of the alkylating agents (e.g. chlorambucil, cyclophosphamide, melphalan), ionizing radiation (causes DNA breaks) and cisplatin (causes DNA cross-link) can cause prolonged azoospermia; nitrosoureas can cause 
azoospermia in adulthood when used for treatment prior to puberty; while other agents cause permanent or prolonged azoospermia only when applied in combination with other gonadotoxic agents (e.g busulfan). ${ }^{6}$ Despite the fact that spermatogenesis can be reinstated after prolonged azoospermia and semen quality can be reduced 7,8 , it doesn't seem that intensified chemotherapy is the most important predictor for reducing fertility. ${ }^{9}$ It is therefore crucial that we explain the possible negative impact of oncological treatment on fertility, and offer the possibility of fertility preservation to male cancer patients prior to treatment. ${ }^{10}$ Data in literature show that semen cryopreservation before the start of oncological treatment is a highly successful method for male fertility preservation. ${ }^{11,12}$ This method can be used from puberty onwards. It has been shown that the success rate of in vitro fertilization (IVF) procedures in healthy men is comparable when fresh or cryopreserved semen is used. ${ }^{13,14}$ Before puberty, only testicular tissue can be cryopreserved for later re-transplantation or in vitro manipulation after the end of treatment. ${ }^{15}$ This procedure, however, is still experimental and has only been successfully used in animal models ${ }^{16,17}$ and monkeys. ${ }^{18}$

The aim of the present study was to retrospectively analyze the success rates of IVF procedures that were performed with the semen of men who had been treated for cancer in the past. We determined whether there are differences in IVF/intracytoplasmic sperm injection (IVF/ICSI) success rates when fresh or cryopreserved semen is used.

\section{Patients and methods}

We performed a retrospective analysis of IVF/ICSI cycles in couples where the male partner had previously been treated for cancer. All consecutive cycles performed at our IVF center at the University Medical Centre in Ljubljana, in the period between January 2004 and December 2018, were included. First, we analyzed all cycles together, regardless of whether fresh or frozen semen was used for oocyte fertilization. We then divided the couples into two groups: one group in which cryopreserved semen was used for oocyte fertilization, and one group where fresh semen, obtained on the day of oocyte aspiration, was used. In all cases included in this retrospective study, the semen was cryopreserved before oncological treatment. Cryopreserved semen was used for IVF/ICSI in all azoospermic and aspermic patients. Fresh semen was used when the semen was of proper quality, so that high fertilization rates were expected. The decision on which sperm to use was made for each patient/sperm sample individually. If fertilization rates in cycles using fresh semen were low, frozen thawed semen was used in the next IVF/ICSI cycle. Similarly, if the development of embryos was poor in the previous cycle, we changed the type of semen to be used for the next IVF/ICSI procedure, where possible. Fresh semen was also used in patients where semen was not cryopreserved prior to oncological treatment.

In the majority of cases, embryo transfer was performed on day 5 . However, in cases where there were only 1 or 2 embryos available for ET, or there was poor embryo development until day 5 in the previous cycle, embryos were transferred on day 3. In cases of poor embryo development, the rest of the embryos were cultured until day 5/6, and cryopreserved if developed to blastocysts of appropriate quality, according to Gardner et al. ${ }^{19}$ The same criteria for the cryopreservation of supernumerary embryos were considered when all embryos were cultured until day 5/6.

To determine the differences between the groups, data were analyzed by using Pearson's chi-square test, Fisher's exact test and a Student's t-test. A p value of less than 0.05 was considered as statistically significant.

The study did not have to be notified in the Ethics Committee according to Slovene law, as it was a register-based study where all the participants signed individual personal approval and permission before starting the treatment (Personal Data Protection Act, Official Gazette of the Republic of Slovenia No 94/07, 2004). Additionally, by Slovenian law, the healthcare providers are obligated to collect data about assisted reproduction procedures and follow the success rates (Healthcare Databases Act, Official Gazette of the Republic of Slovenia No 65/00, 2000; No 47/15, 2015; 31/18, 2018).

\section{Results}

We retrospectively analyzed the outcome of IVF/ ICSI cycles where cryopreserved or fresh semen from male oncological patients was used for oocyte fertilization. Table 1 presents types of cancer, and the number of cases for each cancer type in the cryopreserved and fresh semen groups. There were 214 such IVF/ICSI cycles performed in 115 couples between January 2004 and December 2018. This represents $1.5 \%$ of all cycles performed during this 
period. The mean female age was $31.9 \pm 4.6$ years. Altogether, 2,102 oocytes were retrieved $(9.8 \pm 5.8$ per cycle). After fertilization (the results are for conventional IVF and ICSI together), 1,007 oocytes (47.9\%) were fertilized, 363 (17.3\%) were immature, $262(12.5 \%)$ degenerated and $26(1.2 \%)$ were incorrectly fertilized (polyploidies). Nine hundred and sixty-three fertilized oocytes (95.6\%) developed into embryos, resulting in $4.5 \pm 3.6$ embryos per cycle. On the third day after oocyte aspiration, 63 (6.5\%) of the embryos were transferred to the uterus, and the remaining embryos $(900 ; 93.5 \%)$ were cultured until the fifth or sixth day of development. Of these embryos, 289 (32.1\%) developed to blastocysts, and at least one blastocyst was obtained in 121 IVF/ICSI cycles (56.5\%). Altogether, fresh embryo transfer (ET) was performed in 176 cycles; in 32 cycles $(15.0 \%)$ there were no embryos of appropriate quality for transfer. Cryopreservation of all embryos was conducted in 6 cycles. Supernumerary embryos were cryopreserved $(148 ; 15.4 \%)$ and cryopreservation was performed in 55 cycles (25.7\%). Embryo utilization rate (the number of transferred embryos plus the number of cryopreserved) was $45.2 \%$. Pregnancy rate per ET (with mean number
TABLE 1. Types of cancer in male patients, and the number of cases for each cancer type in the cryopreserved and fresh semen groups

\begin{tabular}{lcc}
\hline & $\begin{array}{c}\text { Cryopreserved } \\
\text { semen (N) }\end{array}$ & $\begin{array}{c}\text { Fresh } \\
\text { semen(N) }\end{array}$ \\
\hline Testicular cancer & 39 & 36 \\
\hline Hodgkin lymphoma & 16 & 7 \\
\hline Non-Hodgkin lymphoma & 0 & 1 \\
\hline Burkitt lymphoma & 1 & 0 \\
\hline Leukemia & 7 & 5 \\
\hline Plasmacytoma & 3 & 1 \\
\hline Rectal cancer & 1 & 2 \\
\hline Brain tumor & 1 & 0 \\
\hline Malignant melanoma & 1 & 0 \\
\hline Prostate cancer & 1 & 0 \\
\hline Epipharynx tumor & 0 & 1 \\
\hline Together & 70 & 53 \\
\hline
\end{tabular}

of transferred embryos $1.6 \pm 0.5$ ) was $32.4 \%$ and $26.6 \%$ per oocyte aspiration. Miscarriage occurred in 12 pregnancies (21.1\%). There were 44 deliveries

TABLE 2. The baseline characteristics of patients and in vitro fertilization / intracytoplasmic sperm injection (IVF/ICSI) cycles. Statistically significant differences are marked with an asterisk ( $p$ value $<0.05$ )

\begin{tabular}{|c|c|c|c|}
\hline & Cryopreserved semen & Fresh semen & $p$ values \\
\hline \multicolumn{4}{|l|}{ Causes of female infertility } \\
\hline Tubal factor & $2(1.5 \%)$ & $1(1.2 \%)$ & 1 \\
\hline Endometriosis & $2(1.5 \%)$ & $2(2.4 \%)$ & 1 \\
\hline Endocrine disorders & $16(12.3 \%)$ & $2(2.4 \%)$ & $0.011^{*}$ \\
\hline Uterine factor & $9(6.9 \%)$ & $11(13.1 \%)$ & 0.13 \\
\hline Cervical factor & 0 & $5(6.0 \%)$ & $0.009^{*}$ \\
\hline Multiple causes of female infertility & $15(11.5 \%)$ & $20(23.8 \%)$ & $0.018^{*}$ \\
\hline No cause of female infertility & $86(66.2 \%)$ & $43(51.2 \%)$ & $0.029^{*}$ \\
\hline \multicolumn{4}{|l|}{ Ovarian stimulation protocols } \\
\hline Long agonist protocol & $74(56.9 \%)$ & $53(63.1 \%)$ & 0.37 \\
\hline Short antagonist protocol & $55(42.3 \%)$ & $30(35.7 \%)$ & 0.33 \\
\hline Others & $1(0.8 \%)$ & $1(1.2 \%)$ & 1 \\
\hline Number of cycles & 130 & 84 & \\
\hline Number of couples & 71 & 53 & \\
\hline Female mean age $( \pm S D)$ & $31.7 \pm 4.8$ & $32.3 \pm 4.3$ & 0.31 \\
\hline Number and rate of ICSI cycles & $122(93.8 \%)$ & $40(84.5 \%)$ & $0.025^{*}$ \\
\hline Oocyte number (mean number per cycle) & $1273(9.8 \pm 5.2)$ & $829(9.9 \pm 6.7)$ & 0.93 \\
\hline Number of MII oocytes (ICSI cycles only) & 974 & 568 & \\
\hline
\end{tabular}

MII = metaphase $\|$ 
TABLE 3. The outcome of the in vitro fertilization / intracytoplasmic sperm injection (IVF/ICSI) cycles in terms of oocytes and embryos according to the type of semen used. Statistically significant differences are marked with an asterisk ( $p$ value < 0.05$)$

\begin{tabular}{|c|c|c|c|}
\hline & $\begin{array}{l}\text { Cryopreserved } \\
\text { semen }\end{array}$ & Fresh semen & $\mathrm{p}$ value \\
\hline Rate of normally fertilized oocytes (per COC, IVF and ICSI together) & $46.3 \%$ & $50.4 \%$ & 0.06 \\
\hline Rate of normally fertilized oocytes (per Mll oocytes number) & $55.6 \%$ & $59.3 \%$ & 0.16 \\
\hline Number and rate of immature oocytes (\%) & $225(17.7 \%)$ & $138(16.6 \%)$ & 0.54 \\
\hline $\begin{array}{l}\text { Number and rate of degenerated oocytes (\% per COC, IVF and ICSI } \\
\text { together) }\end{array}$ & $171(13.4 \%)$ & $91(11.0 \%)$ & 0.10 \\
\hline Number and rate of degenerated oocytes (\% per MIl oocytes number) & $166(17.0 \%)$ & $88(15.5 \%)$ & 0.43 \\
\hline Number and rate of polyploidies (\% per all COC retrieved) & $13(1.0 \%)$ & $13(1.6 \%)$ & 0.27 \\
\hline Number and proportion of embryos (\% per normally fertilized oocytes) & $560(95.1 \%)$ & $403(96.4 \%)$ & 0.31 \\
\hline Mean number of embryos per cycle (mean number \pm SD) & $4.3 \pm 3.4$ & $4.8 \pm 3.8$ & 0.33 \\
\hline Number of embryos cultured until day $5 / 6$ & $518(92.5 \%)$ & $382(94.8 \%)$ & 0.16 \\
\hline Number and rate of blastocysts (\% per embryos cultured until day $5 / 6$ ) & $177(34.2 \%)$ & $112(29.3 \%)$ & 0.12 \\
\hline Number and rate of embryo utilization (transferred plus frozen embryos) & $274(48.9 \%)$ & $161(40.0 \%)$ & $0.006^{*}$ \\
\hline Number of cycles with at least one blastocyst & $74(56.9 \%)$ & $47(56.0 \%)$ & 0.89 \\
\hline Number and rate of cryopreserved embryos (\% of all embryos) & $97(17.3 \%)$ & $51(12.7 \%)$ & $0.048^{*}$ \\
\hline Number and proportion of cycles with embryo cryopreservation & $39(30.0 \%)$ & $16(19.0 \%)$ & 0.07 \\
\hline Number and proportion of cycles with freezing/without ET & $4(3.1 \%)$ & $2(2.4 \%)$ & 1 \\
\hline Number and proportion of cycles without freezing/without ET & $17(13.1 \%)$ & 15 (17.9\%) & 0.34 \\
\hline
\end{tabular}

$\mathrm{COC}=$ cumulus oocyte complex; $\mathrm{ET}=$ embryo transfer; $\mathrm{MII}=$ metaphase $\|$

leading to a $25.0 \%$ live-birth rate per ET and $20.6 \%$ per oocyte aspiration. Twins were born in three cases, representing $6.8 \%$ of all births.

Due to the nature of the oncological disease and its treatment, it is sometimes necessary to use the semen that has been cryopreserved prior to the start of the treatment in IVF/ICSI procedures. Therefore, we further analyzed whether there was a difference in the outcome of IVF/ICSI cycles, depending on whether fresh or cryopreserved semen was used. We summarized the types of cancer in male patients and the number of cases in Table 1 , while the baseline characteristics of analyzed IVF/ ICSI cycles are presented in Table 2. In the analyzed period, cryopreserved semen was used in 130 IVF/ ICSI cycles, and fresh semen, obtained on the day of oocytes aspiration, was used in 84 IVF/ICSI cycles. Detailed results of these procedures are shown in Tables 3 and 4. Briefly, the cryopreserved semen and fresh semen groups were comparable for mean female's age $(31.7 \pm 4.8 v$ vs. $32.3 \pm 4.3)$, mean number of oocytes retrieved per aspiration $(9.8 \pm 5.2$ vs. $9.9 \pm$ 6.7), fertilization rate per retrieved cumulus-oocyte complexes ( $46.3 \%$ vs. $50.4 \%$ ), and mean number of embryos obtained per cycle ( $4.3 \pm 3.4$ vs. $4.8 \pm 3.8)$. There was a significant difference in some causes of female infertility. Briefly, significantly more women had an endocrinological cause of infertility, and a significantly higher number of couples did not have any female's cause of infertility in the cryopreserved semen group (Table 2). In the fresh semen group, a significantly higher number of women had more than one cause of infertility. In the cryopreserved semen group, a statistically significant higher rate of cryopreserved embryos $(17.3 \%$ vs. $12.7 \% ; p=0.048)$ and a higher embryo utilization rate $(48.9 \%$ vs. $40.0 \%$; $\mathrm{p}=0.006)$ were observed. Furthermore, ICSI was performed more often $(93.8 \%$ vs. $84.5 \%$; $\mathrm{p}=0.025)$ in the cryopreserved semen group. ICSI was performed only in cases of impaired semen quality, (oligo-, astheno-, or teratozoospermia, or a combination of these conditions).

In terms of the clinical outcomes of IVF/ICSI cycles, there were no differences in pregnancy rate per ET ( $35.8 \%$ vs. $26.9 \%)$, live birth rate per fresh ET $(26.6 \%$ vs. $22.4 \%)$ and miscarriage rate per pregnancy $(25.6 \%$ vs. $11.1 \%)$ between the cryopreserved and fresh semen groups. As presented in Table 4, the proportion of day 3 ETs was similar between the analyzed groups. However, when we analyzed cumulative pregnancy rate per couple (fresh and frozen-thawed cycles together), the results showed that at least one pregnancy was achieved in $60.6 \%$ 
TABLE 4. Clinical outcome of in vitro fertilization / intracytoplasmic sperm injection (IVF/ICSI) cycles according to the type of semen used. Statistically significant difference is marked with an asterisk ( $p$ value $<0.05$ )

\begin{tabular}{|c|c|c|c|}
\hline Number of all ETs & 109 & 67 & 0.45 \\
\hline Mean number of transferred embryos ( \pm SD) & $1.6 \pm 0.5$ & $1.6 \pm 0.5$ & 0.81 \\
\hline Number of pregnancies (\% per ET) & $39(35.8 \%)$ & $18(26.9 \%)$ & 0.22 \\
\hline Live births (\% per aspiration) & $29(22.3 \%)$ & $15(17.9 \%)$ & 0.43 \\
\hline Miscarriages & $10(25.6 \%)$ & $\begin{array}{l}2(11.1 \%) \\
(+1 \times \mathrm{EU})\end{array}$ & 0.21 \\
\hline Gestational age (all births) & $38.4 \pm 2.6$ & $36.0 \pm 5.1$ & 0.14 \\
\hline Gestational age for singletons & $38.5 \pm 2.7$ & $36.6 \pm 4.9$ & 0.18 \\
\hline Cumulative number and rate of couples with at least one live birth & $\begin{array}{c}32 \text { (45.1\%) } \\
\text { (+4 ongoing pregnancies) }\end{array}$ & $18(34.0 \%)$ & 0.21 \\
\hline
\end{tabular}

$\mathrm{EU}=$ extrauterine pregnancy; $\mathrm{ET}=$ embryo transfer

of couples in the cryopreserved semen group, and in $37.7 \%$ of couples in the fresh semen group. The difference was statistically significant $(p=0.012)$. Despite this difference, the proportion of couples achieving at least one birth was similar on a cumulative level, with $45.1 \%$ in the cryopreserved group and $34.0 \%$ in the fresh semen group $(\mathrm{p}=0.21)$.

There were 9 couples who underwent 33 cycles (16 cycles with cryopreserved semen and 17 cycles with fresh semen) and used both cryopreserved and fresh semen for the IVF/ICSI procedure. Four couples started the treatment with cryopreserved semen, five with fresh semen, and in 7 of these couples, the treatment led to live birth. One couple had two children, the first one conceived with cryopreserved semen, and second one with fresh semen.

The mean duration of cryopreservation of semen was $6.6 \pm 4.4$ years (minimum 1 year and maximum 19 years), and the mean semen volume per sample prior to cryopreservation was $3.1 \pm 1.5 \mathrm{ml}$. The mean sperm concentration was $31.2 \pm 30.7$ million/ $\mathrm{ml}$, mean sperm motility $34.7 \pm 10.3 \%$, and the proportion of morphologically normal spermatozoa was $16.3 \pm 14.3 \%$. The mean number of cryopreserved straws per cryopreservation procedure was $8.1 \pm 3.8$; however, some patients underwent more than just one cryopreservation procedure, and the mean number of cryopreserved straws per patient was $11.2 \pm 5.6$. Until now, $40.0 \%$ of these samples have been used for IVF/ICSI procedures, and 14.1\% of patients included in this analysis have used all of their cryopreserved semen. After the thawing of the samples, and before using them for the IVF/ ICSI procedure, the mean sperm motility was 30.9 $\pm 19.9 \%$, the proportion of morphologically normal spermatozoa was $20.1 \pm 16.5 \%$, and the mean sperm concentration was $18.8 \pm 22.0$ million $/ \mathrm{ml}$. This concentration is lower than in fresh samples, but this is to be expected because sperm samples are diluted in a 1:1 ratio with freezing solution before cryopreservation. In the group of patients where fresh semen was used, the mean sperm concentration was $34.7 \pm 43.7$ million $/ \mathrm{ml}$, mean sperm motility $50.0 \pm 23.2 \%$, and the proportion of morphologically normal spermatozoa was $16.2 \pm 13.4 \%$.

\section{Discussion}

In the present study, we performed a retrospective analysis of $214 \mathrm{IVF} / \mathrm{ICSI}$ procedures where fresh or cryopreserved semen of men, who were treated for cancer in the past, was used. We found that the success of the IVF/ICSI procedures, in terms of pregnancies and live-births after fresh embryo transfer, was the same regardless of the type of semen used. However, embryo utilization and cryopreservation rates were significantly higher when cryopreserved 
semen was used, which led to a significantly higher cumulative number of couples who achieved at least one pregnancy. Despite this, the difference in cumulative number of live-births was not statistically significant. One reason for this could be in the non-significant higher number of miscarriages in the cryopreserved semen group.

Altogether, success rates of IVF/ICSI procedures in couples where men were treated for cancer in the past were comparable with success rates of IVF/ICSI procedures in the general population of infertile couples treated in our clinic, and also on a European level. Analyses of all IVF/ICSI cycles (combined data for cryopreserved and fresh semen and for IVF and ICSI) have shown that pregnancy rate per oocyte aspiration was $26.6 \%$, which is similar to the pregnancy rate of infertile patients treated in our center in the same time period (2004-2018), 25.5\% respectively, and the latest pregnancy rate reported for the general population in the European IVF Monitoring (EIM) data from 2016. ${ }^{20}$ EIM collects data on the performance of IVF procedures in European Union Member States (https://www.eshre.eu/Data-collection-and-research/ Consortia/EIM). According to EIM data, in 2016, the proportion of pregnancy rate per oocyte aspiration was $28.0 \%$ for classical IVF and $25.0 \%$ for ICSI procedures. $^{20}$

If we compare the remaining results (oocyte fertilization, embryo development) with which we evaluate the success of IVF/ICSI procedures, with criteria that determine the recommended minimum values ${ }^{21}$, we can see that not all of them reach these values. The desired proportion of fertilized oocytes according to these criteria is $65 \%$ for ICSI and $60 \%$ for classical IVF. In the present analysis, where we did not separate the data according to the type of IVF procedure used for oocyte fertilization, the proportion of fertilized oocytes was $47.9 \%$, and when only ICSI was analyzed this proportion was $57.0 \%$. This proportion is similar to the one reported in one of the very rare studies similar to ours, where $49 \%$ of oocytes were fertilized with classical IVF and 51\% with ICSI. ${ }^{22}$ A similar thing is seen with the desired minimum proportion of embryos that develop to blastocysts. In our study, $32.1 \%$ of embryos cultured until day 5/6 developed to blastocysts, which is lower than the desirable $40 \%$. It is important to note, however, that the minimum criteria are determined on the basis of the general population of "healthy" couples attending IVF. In our study, however, semen obtained from male oncological patients was used, and such semen has been shown to be of poorer quality. ${ }^{23,24}$
Therefore, the observed differences in our study are somewhat expected and most likely cannot be considered significant. In addition to the negative impact of the disease itself or oncological gonadotoxic treatment on the basic criteria of semen quality (number, motility, morphology), the negative impact of the disease is also reflected in increased sperm DNA fragmentation. ${ }^{25,26}$ Meta-analyses have shown that increased sperm DNA fragmentation negatively affects IVF/ICSI outcomes even in the healthy male population. ${ }^{27,28}$

Since a part of the IVF/ICSI procedures was performed with cryopreserved semen which was stored before oncological treatment, and a part of the procedures with fresh semen obtained on the day of oocyte aspiration, after oncological treatment was completed, we checked whether there were any differences in the success rates of IVF procedures according to the type of semen used. The literature shows that the success of IVF/ICSI procedures in healthy men is the same regardless of the type of semen (fresh, cryopreserved) used..$^{13,14}$ However, relatively little data are available in men who have been treated for cancer. In part, the problem is that a relatively small proportion of men who cryopreserve semen before treatment also use it later. It is estimated that semen is used by $1.5 \%$ to $16.3 \%$ of men..$^{11,22,29-35}$ Studies by van Casteren et al. ${ }^{11}$ and Botchan et al..$^{30}$ showed that the proportion of births (per oocyte aspiration) after the use of cryopreserved semen in cancer patients was $24 \%$ and $25 \%$, respectively, which is similar to our results (22.3\%). The study of van Casteren et al..$^{11}$ states that in $20 \%$ of IVF/ICSI procedures no suitable embryo was obtained for transfer. This is more than in our study, where in $15.0 \%$ of procedures (cryopreserved and fresh semen) there were no embryos suitable for transfer. There are also studies that report higher ( $29 \%$ for IVF and $32 \%$ for ICSI) ${ }^{22}$ and much higher $(50 \%)^{36}$ percentage of births per oocyte aspiration. The reason for such a large difference with our results may be in the higher average number of embryos transferred per transfer. At our department, a maximum of two embryos are transferred to the uterus at the same time. In the study of Hourvitz et al. ${ }^{36}$, the average number of embryos transferred to the uterus was $3.0 \pm 1.1$, which is significantly more than in our study $(1.6 \pm 0.5)$.

Interestingly, as mentioned above, our data have shown that in the group of couples where cryopreserved semen was used, embryo utilization and cryopreservation rates were significantly higher compared to couples where fresh semen was used. This finding implies that semen qual- 
ity deteriorates after the oncological treatment. Data of currently published studies, however, are conflicting. Trottmann et al. ${ }^{37}$ stated that semen quality deteriorated drastically after oncological treatment. Weibring et al. ${ }^{38}$ reported similar observations, but further noted that semen quality improves 12 months after the end of treatment. Di Bisceglie et al..$^{39}$ also found that semen quality starts to improve 18 months after the end of treatment. In addition, there is evidence of an improvement of sperm DNA fragmentation after the end of some oncological treatments. ${ }^{40}$ Despite these data, it is highly recommended that semen is cryopreserved in all oncological patients before treatment, even in those undergoing low-risk gonadotoxic treatment. This way, semen of better quality can be chosen to perform IVF/ICSI, if needed.

In the present study, a significantly higher number of women in the cryopreserved semen group did not have any cause of infertility, and IVF was only needed due to the male factor. One would assume that this is the cause of the significantly higher embryo cryopreservation rates in the cryopreserved semen. However, analysis of 121,744 women undergoing their first IVF/ICSI cycles has shown that IVF/ICSI success rates are most significantly affected by female's age and not by female's cause of infertility. ${ }^{41}$ No significant difference in women's age was found in the present study.

As this is a retrospective study, there are some limitations in our data. We included cycles from a long time period, and some changes in our work have been introduced into clinical practice since then. For instance, in 2004, long GnRH agonist protocol was mostly used for controlled ovarian hyperstimulation, and it has been mostly replaced with short GnRH antagonist protocol since 2010. There have also been some changes in embryo culture approach, and embryos have been cultured in lower oxygen concentration (5\%) since 2008. Prior to 2008, they were cultured in an atmospheric concentration of oxygen (21\%). Additionally, there have been a few changes of manufacturers and types of embryo culture media. Despite these limitations, this shouldn't impair the conclusions drawn from our results, as the described changes were applied for all couples (cryopreserved and fresh semen groups).

\section{Conclusions}

Semen cryopreservation before the start of oncological treatment is a widely accepted and successful method to maintain the fertility of men with cancer, as oncological treatment can negatively affect spermatogenesis. The results of our retrospective study show that the results of IVF/ICSI procedures in terms of pregnancies and live-birth rates after fresh embryo transfer are comparable regardless of whether we use fresh or cryopreserved semen from male cancer patients. Additionally, while the embryo utilization and embryo cryopreservation rates are higher if cryopreserved semen is used, this leads to a higher cumulative pregnancy rate and possibly to a higher cumulative birth rate. Overall results also show that the success rates of IVF/ICSI procedures where fresh or cryopreserved semen of male cancer patients was used are comparable with the results of IVF/ICSI procedures in the general population of infertile couples at European level, as well as with the population of couples treated at our clinic in the same time period.

\section{Acknowledgments}

The authors would like to thank all gynecologists, clinical embryologists, medical nurses and other staff at the Department of Human Reproduction, Division of Gynaecology, University Medical Centre Ljubljana, Slovenia, for their support.

\section{References}

1. Ragheb AM, Sabanegh ES Jr. Male fertility-implications of anticancer treatment and strategies to mitigate gonadotoxicity. Anticancer Agents Med Chem 2010; 10: 92-102. doi: 10.2174/1871520611009010092

2. Howell SJ, Shalet SM. Testicular function following chemotherapy. Hum Reprod Update 2001; 7: 363-9. doi: 10.1093/humupd/7.4.363

3. Johnson MD, Cooper AR, Jungheim ES, Lanzendorf SE, Odem RR, Ratts VS Sperm banking for fertility preservation: a 20-year experience. Eur J Obstet Gynecol Reprod Biol 2013; 170: 177-82. doi: 10.1016/j.ejogrb.2013.06.021

4. Reebals JF, Brown R, Buckner EB. Nurse practice issues regarding sperm banking in adolescent male cancer patients. J Pediatr Oncol Nurs 2006; 23 : 182-8. doi: $10.1177 / 1043454206289868$

5. Giwercman A, Petersen PM. Cancer and male infertility. Baillieres Best Practice Res Clin Endo Metab 2000; 14: 453-71. doi: 10.1053/ beem.2000.0091

6. Meistrich ML. Effects of chemotherapy and radiotherapy on spermatogenesis in humans. Fertil Steril 2013; 100: 1180-6. doi: 10.1016/j.fertnstert.2013.08.010

7. Neal MS, Nagel K, Duckworth J, Bissessar H, Fischer MA, Portwine C, et al. Effectiveness of sperm banking in adolescents and young adults with cancer: a regional experience. Cancer 2007; 110: 1125-9. doi: 10.1002/ cncr.22889

8. Tournaye H, Goossens E, Verheyen G, Frederickx V, De Block G, Devroey P, et al. Preserving the reproductive potential of men and boys with cancer: current concepts and future prospects. Hum Reprod Update 2004; 10: 525-32. doi: 10.1093/humupd/dmh038

9. Boltežar L, Pintarić K, Jezeršek Novaković B. Fertility in young patients following treatment for Hodgkin's lymphoma: a single center survey. J Assist Reprod Genet 2016; 33: 325-33. doi:10.1007/s10815-015-0636-6 
10. Loren AW, Mangu PB, Beck LN, Brennan L, Magdalinski AJ, Partridge AH et al. Fertility preservation for patients with cancer: American Society of Clinical Oncology clinical practice guideline update. J Clin Oncol 2013; 31: 2500-10. doi: 10.1200/JCO.2013.49.2678

11. van Casteren NJ, van Santbrink EJ, van Inzen W, Romijn JC, Dohle GR. Use rate and assisted reproduction technologies outcome of cryopreserved semen from 629 cancer patients. Fertil Steril 2008; 90: 2245-50. doi 10.1016/j.fertnstert.2007.10.055

12. Depalo R, Falagario D, Masciandaro P, Nardelli C, Vacca MP, Capuano P, et al. Fertility preservation in males with cancer: 16-year monocentric experience of sperm banking and post-thaw reproductive outcomes. Ther Adv Med Oncol 2016; 8: 412-20. doi: 10.1177/1758834016665078

13. Englert $Y$, Delvigne A, Vekemans $M$, Lejeune B, Henlisz A, de Maertelaer $G$, et al. Is fresh or frozen semen to be used in in vitro fertilization with dono sperm? Fertil Steril 1989; 51: 661-4. doi: 10.1016/S0015-0282(16)60617-9

14. Eastick J, Venetis C, Cooke S, Storr A, Susetio D, Chapman M. Is early embryo development as observed by time-lapse microscopy dependent on whether fresh or frozen sperm was used for ICSI? A cohort study. J Assist Reprod Genet 2017; 34: 733-40. doi: 10.1007/s10815-017-0928-0

15. Tournaye $\mathrm{H}$, Dohle GR, Barratt CL. Fertility preservation in men with cancer. Lancet 2014; 384: 1295-301. doi: 10.1016/S0140-6736(14)60495-5

16. Honaramooz A, Snedaker A, Boiani M, Schöler H, Dobrinski I, Schlatt S. Sperm from neonatal mammalian testes grafted in mice. Nature 2002; 418 : 778-81. doi: 10.1038/nature00918

17. Rodriguez-Sosa JR, Dobrinski I. Recent developments in testis tissue xenografting. Reproduction 2009; 138: 187-94. doi: 10.1530/REP-09-0012

18. Fayomi AP, Peters K, Sukhwani M, Valli-Pulaski H, Shetty G, Meistrich ML, et al. Autologous grafting of cryopreserved prepubertal rhesus testis produces sperm and offspring. Science 2019; 363: 1314-9. doi: 10.1126/science. aav2914. Erratum in: Science 2019; 364: eaax4999. doi: 10.1126/science. aax4999

19. Gardner DK, Lane M, Stevens J, Schlenker T, Schoolcraft WB. Blastocyst score affects implantation and pregnancy outcome: towards a single blastocyst transfer. Fertil Steril 2000; 73: 1155-8. doi: 10.1016/s00150282(00)00518-5

20. European IVF-monitoring Consortium (EIM) for the European Society of Human Reproduction and Embryology (ESHRE), Wyns C, Bergh C, Calhaz Jorge C, De Geyter Ch, Kupka MS, Motrenko T, et al. ART in Europe, 2016 results generated from European registries by ESHRE. Hum Reprod Open 2020; 2020: hoaa032. doi: 10.1093/hropen/hoaa032

21. ESHRE Special Interest Group of Embryology and Alpha Scientists in Reproductive Medicine. The Vienna consensus: report of an expert meeting on the development of ART laboratory performance indicators. Reprod Biomed Online 2017; 35: 494-510. doi: 10.1016/j.rbmo.2017.06.015

22. Muller I, Oude Ophuis RJ, Broekmans FJ, Lock TM. Semen cryopreservation and usage rate for assisted reproductive technology in 898 men with cancer Reprod Biomed Online 2016; 32: 147-53. doi: 10.1016/j.rbmo.2015.11.005

23. Auger J, Sermondade N, Eustache F. Semen quality of 4480 young cance and systemic disease patients: baseline data and clinical considerations. Basic Clin Androl 2016; 26: 3. doi: 10.1186/s12610-016-0031-x

24. Degl'Innocenti $S$, Filimberti $E$, Magini A, Krausz C, Lombardi G, Fino $M G$, et al. Semen cryopreservation for men banking for oligospermia, cancers, and other pathologies: prediction of post-thaw outcome using basal semen quality. Fertil Steril 2013; 100: 1555-63. doi: 10.1016/j.fertnstert.2013.08.005

25. Tamburrino L, Cambi M, Marchiani S, Manigrasso I, Degl'Innocenti S, Fort $\mathrm{G}$, et al. Sperm DNA fragmentation in cryopreserved samples from subjects with different cancers. Reprod Fertil Dev 2017; 29: 637-45. doi: 10.1071/ RD15190

26. Said TM, Tellez S, Evenson DP, Del Valle AP. Assessment of sperm quality, DNA integrity and cryopreservation protocols in men diagnosed with testicular and systemic malignancies. Andrologia 2009; 41: 377-82. doi: 10.1111/j.1439-0272.2009.00941.x

27. Osman A, Alsomait $H$, Seshadri S, El-Toukhy T, Khalaf $Y$. The effect of sperm DNA fragmentation on live birth rate after IVF or ICSI: a systematic review and meta-analysis. Reprod Biomed Online 2015; 30: 120-7. doi: 10.1016/j. rbmo.2014.10.018
28. Deng C, Li T, Xie Y, Guo Y, Yang QY, Liang X, et al. Sperm DNA fragmentation index influences assisted reproductive technology outcome: a systematic review and meta-analysis combined with a retrospective cohort study. Andrologia 2019; 51: e13263. doi: 10.1111/and.13263

29. Machen GL, Harris SE, Bird ET, Brown ML, Ingalsbe DA, East MM, et al. Utilization of cryopreserved sperm cells based on the indication for storage. Investig Clin Urol 2018; 59: 177-81. doi: 10.4111/icu.2018.59.3.177

30. Botchan A, Karpol S, Lehavi O, Paz G, Kleiman SE, Yogev L, et al. Preservation of sperm of cancer patients: extent of use and pregnancy outcome in a tertiary infertility center. Asian J Androl 2013; 15: 382-6. doi: 10.1038/ aja.2013.3

31. Lass A, Akagbosu F, Brinsden P. Sperm banking and assisted reproduction treatment for couples following cancer treatment of the male partner. Hum Reprod Update 2001; 7: 370-7. doi: 10.1093/humupd/7.4.370

32. Kelleher S, Wishart SM, Liu PY, Turner L, Di Pierro I, Conway AJ, et al. Longterm outcomes of elective human sperm cryostorage. Hum Reprod 2001; 16: 2632-9. doi: 10.1093/humrep/16.12.2632

33. Agarwal A, Ranganathan P, Kattal N, Pasqualotto F, Hallak J, Khayal S, et al Fertility after cancer: a prospective review of assisted reproductive outcome with banked semen specimens. Fertil Steril 2004; 81: 342-8. doi: 10.1016/j. fertnstert.2003.07.021

34. Meseguer M, Molina N, García-Velasco JA, Remohí J, Pellicer A, Garrido N. Sperm cryopreservation in oncological patients: a 14-year follow-up study. Fertil Steril 2006; 85: 640-5. doi: 10.1016/j.fertnstert.2005.08.022

35. Ragni G, Somigliana E, Restelli L, Salvi R, Arnoldi M, Paffoni A. Sperm banking and rate of assisted reproduction treatment: insights from a 15-year cryopreservation program for male cancer patients. Cancer 2003; 97: 1624-9. doi: 10.1002/cncr.11229

36. Hourvitz A, Goldschlag DE, Davis OK, Gosden LV, Palermo GD, Rosenwaks Z. Intracytoplasmic sperm injection (ICSI) using cryopreserved sperm from men with malignant neoplasm yields high pregnancy rates. Fertil Steril 2008; 90: 557-63. doi: 10.1016/j.fertnstert.2007.03.002

37. Trottmann M, Becker AJ, Stadler T, Straub J, Soljanik I, Schlenker B, et al. Semen quality in men with malignant diseases before and after therapy and the role of cryopreservation. Eur Urol 2007; 52: 355-67. doi: 10.1016/j. eururo.2007.03.085

38. Weibring K, Nord C, Ståhl O, Eberhard J, Sandberg K, Johansson H, et al. Sperm count in Swedish clinical stage I testicular cancer patients following adjuvant treatment. Ann Oncol 2019; 30: 604-11. doi: 10.1093/annonc/ mdz017

39. Di Bisceglie C, Bertagna A, Composto ER, Lanfranco F, Baldi M, Motta G, et al. Effects of oncological treatments on semen quality in patients with testicular neoplasia or lymphoproliferative disorders. Asian J Androl 2013; 15: 425-9. doi: 10.1038/aja.2012.171

40. Smit M, van Casteren NJ, Wildhagen MF, Romijn JC, Dohle GR. Sperm DNA integrity in cancer patients before and after cytotoxic treatment. Hum Reprod 2010; 25: 1877-83. doi: 10.1093/humrep/deq104

41. Bhattacharya S, Maheshwari A, Mollison J. Factors associated with failed treatment: an analysis of 121,744 women embarking on their first IVF cycles. PLoS One 2013; 8: e82249. doi: 10.1371/journal.pone.0082249 